\title{
Small Total Dose Measurement System for SOHLA-1 and SDS-1
}

\author{
Yugo KIMOTO ${ }^{1)}$, Yohei SATOH ${ }^{1)}$ and Hiroshi TACHIHARA ${ }^{1)}$ \\ 1) Aerospace Research and Development Directorate, Japan Aerospace Exploration Agency (JAXA), Tsukuba, Japan
}

(Received May 2nd, 2008)

\begin{abstract}
The Japanese Aerospace Exploration Agency (JAXA) uses monitors on board satellites to measure and record in-flight data about ionization effects in space. A compact, total-dose measurement system for small satellites-Space-Oriented Higashiosaka Leading Association -1 (SOHLA-1) and Small Demonstration-Satellite -1 (SDS-1) — was developed based on a prior system for measuring total ionizing dose effects. Especially, the sensor for SDS-1 is much smaller than the sensor for SOHLA-1. The sensor for SDS-1 is $8 \mathrm{~mm}$ wide $\times 3 \mathrm{~mm}$ high $\times 19 \mathrm{~mm}$ long and weighs approximately $4 \mathrm{~g}$ with $500 \mathrm{~mm}$ with its wire harness. An 8-pin Lead less Chip Carrier (LCC) RADFET and temperature sensor are arranged on it. Seven sensors are mounted on some components inside the SDS-1. The sensor for SOHLA-1 is a 14-pin Dual Inline Package (DIP) type RADFET. The four sensors, which have RADFET on a printed board covered with an aluminum chassis, are mounted both inside and outside the satellite. This report presents small total dose measurement systems and ground irradiation test results for two small satellites.
\end{abstract}

Key Words: Total dose, RADFET, SOHLA-1, SDS-1

\section{Nomenclature}

$d V g s \quad$ : Difference of Vgs (the voltage at the source with the drain/gate grounded ) from the initial value

\section{Introduction}

National Space Development Agency (NASDA), the forerunner of the Japanese Aerospace Exploration Agency (JAXA), has measured in-flight space environments and their effects. Data were received from monitors that had been placed aboard satellites. Total ionizing dose effects can degrade microelectronics because of the high energy levels of electrons and protons. The ETS-V satellite, which was launched on 27 August 1987 in geosynchronous orbit (GEO) and which ceased operations on 12 September 1997, had a system for monitoring the degradation of integrated circuits (ICs) (CMOS technology 4049 and TTL technology 54LS04) according to the total dose. It produced threshold voltage (Vth) degradation data for its 10 active years; the data indicated a correlation between Vth degradation and high-energy electrons in GEO during the solar-minimum period ${ }^{1)}$.

On the Tsubasa (MDS-1) satellite, which flew in a highly eccentric orbit from February 2002 to September 2003, 56 compact total dose sensors were mounted on several experimental modules. The satellite's missions were to verify the functionality of commercial parts and new technologies in bus-system components in space, including a solid-state data recorder, a parallel computer system, and commercial semiconductor devices, and to record ionization-dose measurements in space ${ }^{2)}{ }^{3)}$. A small dosimeter, utilizing Radiation-Sensitive Field Effect Transistors (RADFET) ${ }^{4}$, measured the total dose. These sensors were arranged among the electrical components to measure the total doses.
Small total dose measurement systems for small satellites SOHLA-1 and SDS-1 were developed based on a prior total ionizing dose effect measurement system. A small satellite was developed for the Space Oriented Higashiosaka Leading Association (SOHLA). For the project, JAXA provides technological assistance. The satellite is a W500 mm $\times$ H500 $\mathrm{mm} \times$ D500 mm, 50-kg-class spin-stabilized demonstration satellite. Some mission components, such as novel solar cells, have been demonstrated. Another small satellite is the Small Demonstration Satellite-1 (SDS-1) developed by JAXA; it is the first platform for short-term, low-cost demonstration of advanced technologies. The SDS-1 is of the $100-\mathrm{kg}$ class (actually, $90 \mathrm{~kg}$ ) and is mostly spin-stabilized, but it is triaxially stabilized during experiments. Both satellites will be launched in FY2008 aboard the H-IIA vehicle.

This paper presents the system concept and radiation test results for two small satellites.

\section{Total Dose Measurement System for SOHLA-1 ${ }^{5)}$}

The small satellite, SOHLA-1, is a $500 \mathrm{~mm}$ wide $\times 500$ $\mathrm{mm}$ high $\times 500 \mathrm{~mm}$ long, $50 \mathrm{~kg}$ class, spin-stabilized, demonstration satellite. Figure 1 depicts SOHLA-1, showing some of its mission components ${ }^{6}$.

A total dose measurement system is one mission component. It consists of a RADFET Control Circuit (RCC) and four RADFETs (RFET). Figure 2 depicts the system diagram of the total dose measurement system.

The RCC is a printed board that is covered and attached to an Advanced Microprocessing In-orbit (AMI) experiment equipment component, a 200 MIPS class 64 bit MPU for space application on a $120 \mathrm{~mm}$ wide $\times 50 \mathrm{~mm}$ high $\times 70 \mathrm{~mm}$ long aluminum plate (Fig. 3). Its maximum weight is $0.66 \mathrm{~kg}$. Because the system resources are limited, the RCC interfaces with the satellite system through the AMI for telemetry, 
command, and power. The power consumption of the AMI and $\mathrm{RCC}$ is less than $4 \mathrm{~W}$; that of the RCC alone is $1 \mathrm{~W}$. The RCC requires levels of $+5 \mathrm{~V},+15 \mathrm{~V}$ and $-15 \mathrm{~V}$. The telemetry data interfaces with the AMI at the CMOS 5.0 V-level protocol.

The RCC has one RADFET, a specially designed positive channel metal oxide semiconductor (PMOS) transistor with a thick gate oxide, which is optimized for increased radiation sensitivity. The RADFET is suitable for space use in terms of cost, weight, and low power consumption. The RADFET used in SOHLA-1 is a $400 \mathrm{~nm}$ implanted-gate oxide device manufactured by the Tyndall National Institute in Ireland. The RFET is a printed board on which RADFETs are arranged on an aluminum chassis. It is $47 \mathrm{~mm}$ wide $\times 22 \mathrm{~mm}$ high $\times 39 \mathrm{~mm}$ long and weighs $0.06 \mathrm{~kg}$ without its wire harness (Fig. 4). One RFET (RFET1) is attached outside the satellite wall; another (RFET2) is attached inside the satellite wall. Actually, RFET3 is attached in the center of the satellite. RFET4, without the aluminum chassis, is mounted on the printed board of the RCC

The RADFET in the RCC and the RFET can be connected in one of two modes (Fig. 5). In exposure mode, all pins are grounded during exposure. In measurement mode, the RADFET source/bulk is connected to a constant current (10 $\mu \mathrm{A})$, and the voltage $\left(V_{g s}\right)$ at the source with the drain/gate grounded is measured for $20 \mathrm{~s}$. The measurement mode is used only for AMI experiments. In fact, $V_{g s}$ is measured from 0 to 5 $\mathrm{V}$ with 12-bit resolution.

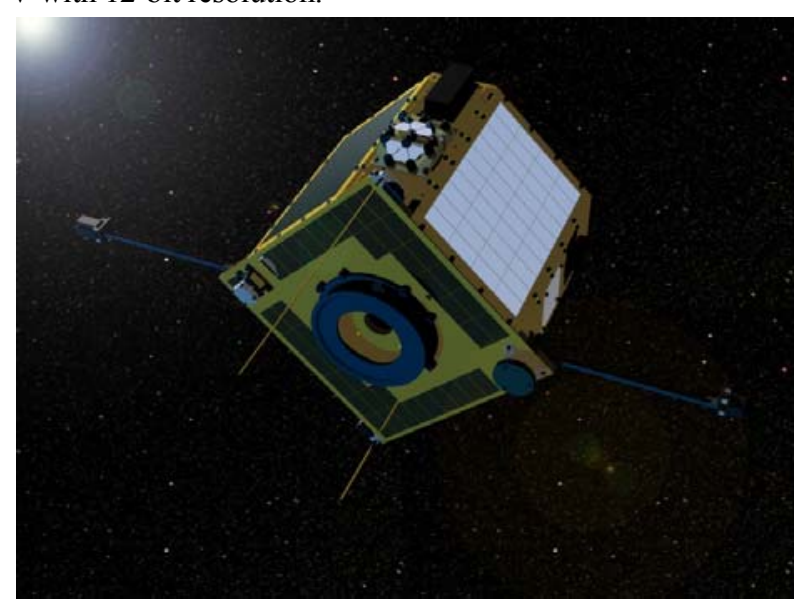

Fig. 1. On-orbit image of SOHLA-1.

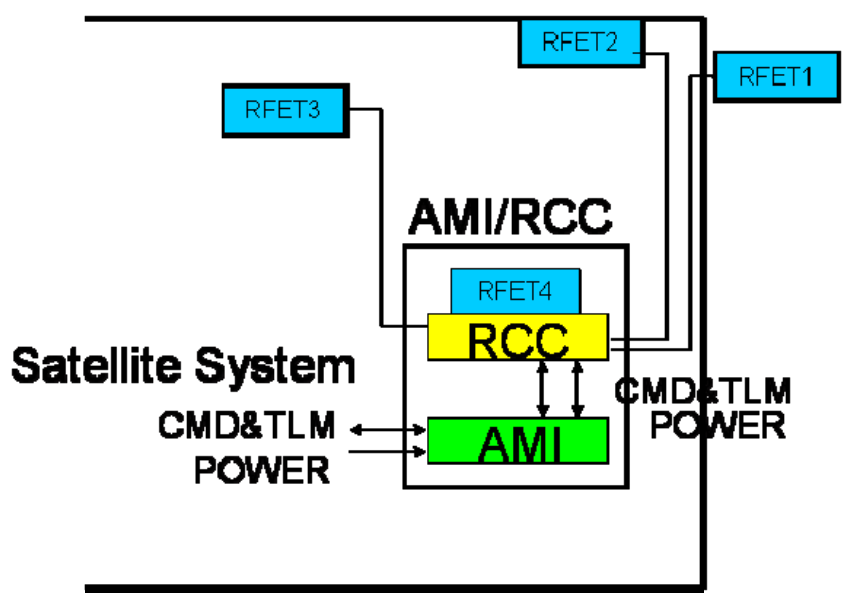

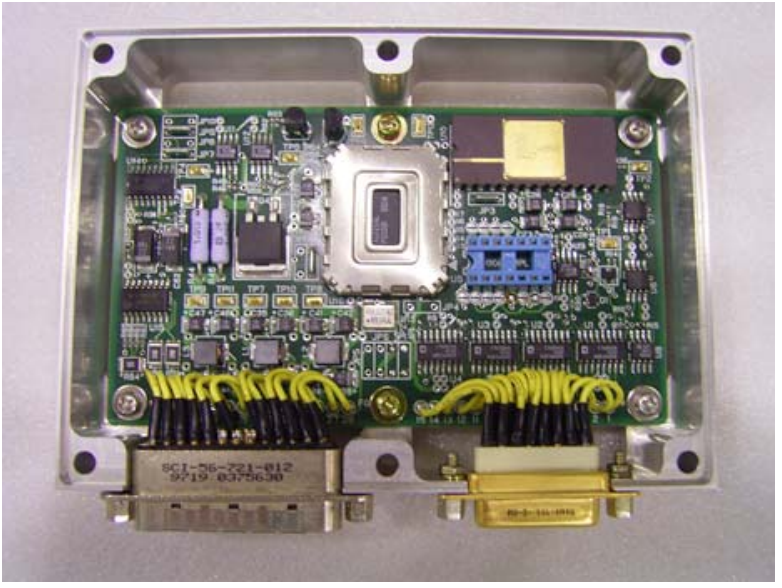

Fig. 3. Photograph of the BBM of an RCC, showing only a printed RCC board.

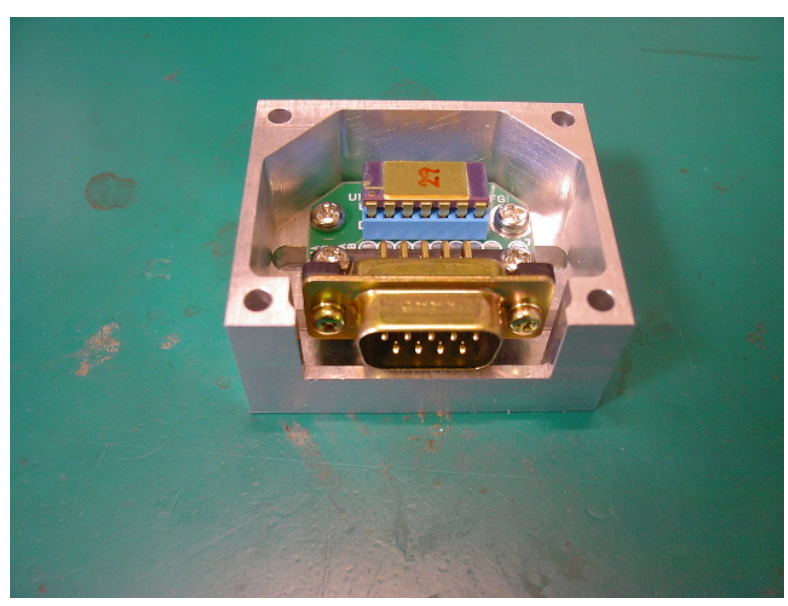

Fig. 4. Photograph of an RFET.
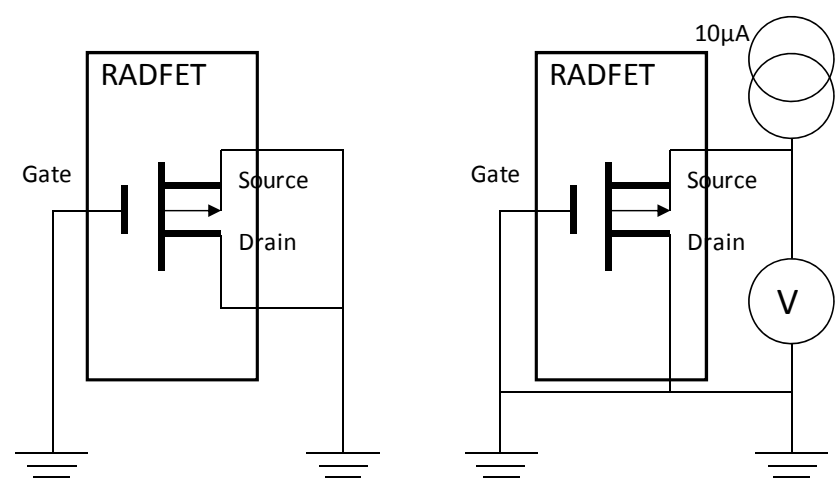

Exposure mode

Measurement mode

Fig. 5. Two modes of RADFET.

The absorbed dose at which the RFETs are arranged is evaluated before flight. This evaluation uses FASTRAD ${ }^{7)}$, a tool for realization of radiation models including CAD. The output from OMERE provides the source data for analyses. Figure 6 presents the CAD image of the satellite wall holding the components: RFET1, RFET3, and RFET4.

Fig. 2. Diagram of the total dose measurement system. 


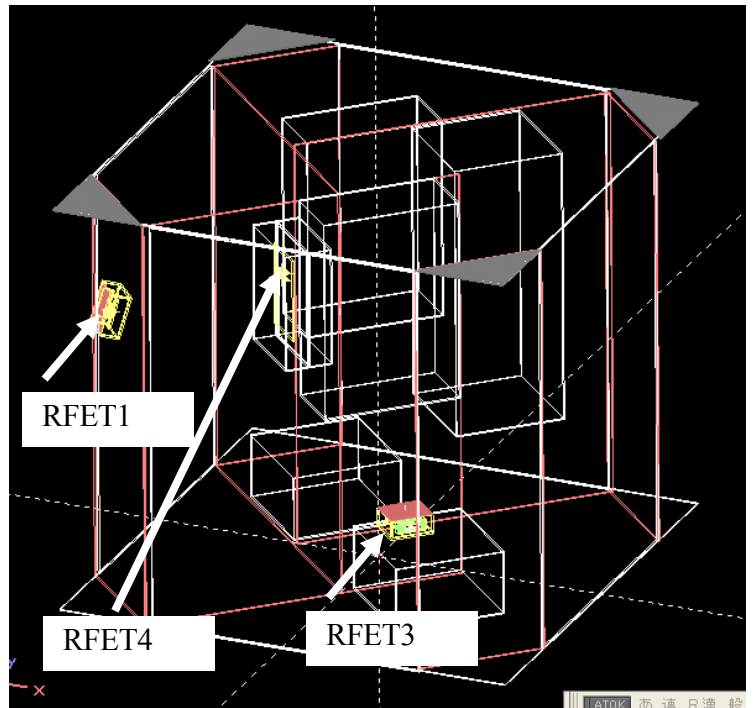

Fig. 6. CAD image of the SOHLA-1 and RFETs.

Table 1 presents calculation results for the absorbed dose and the equivalent aluminum thickness. These results are compared with flight data. The data help direct construction of a highly accurate total dose effect model.

Table 1. Absorbed dose and equivalent aluminum thickness.

\begin{tabular}{llll}
\hline \multicolumn{4}{c}{ Mounting location } \\
\hline & RFET1 & RFET3 & RFET4 \\
\hline Totaldose [Gy] & 4.99 & 1.91 & 2.20 \\
\hline
\end{tabular}

\begin{tabular}{llll}
\multicolumn{4}{l}{ Equivalent aluminum thickness [mm] } \\
\hline Minimum & 2.68 & 3.52 & 4.68 \\
\hline Maximum & 46.85 & 51.66 & 71.59 \\
\hline
\end{tabular}

\section{Total Dose Measurement System for SDS-1}

JAXA's space technology demonstration research center has developed a small, low-cost satellite to demonstrate advanced space technologies in orbit. It has conducted a research and development program for small satellite systems. Small satellite systems are expected to improve efficiency and flexibility, and to secure reliability for new technologies in JAXA's midterm satellite-application strategies. The plan will include two different programs. The Small Demonstration-Satellite (SDS) program is mainly used to demonstrate new technology in JAXA's R\&D activities. The satellite will weigh between 50 and $200 \mathrm{~kg}$ to enable frequent launch opportunities as a piggyback payload. This program is also part of JAXA's reliability and key-technology improvement strategy. The second program will use a 200-400 $\mathrm{kg}$ standard satellite bus for application to the science $\operatorname{program}^{8)}$.

For demonstration processes, quantitative evaluation for tolerance to space radiation is required. Therefore, it is necessary to have a small, lightweight, low-power system for measurement of space radiation effects. Actually, JAXA has developed and flown systems for measuring total ionizing dose effects. A small sensor was developed for the SDS-1 satellite, the first SDS program satellite.

The first SDS series, SDS-1, is a $700 \mathrm{~mm}$ wide $\times 600$ $\mathrm{mm}$ high $\times 700 \mathrm{~mm}$ long, $100 \mathrm{~kg}$ class, spin-stabilized, demonstration satellite. This satellite can be stabilized triaxially when a mission requires it. Figure 7 depicts an orbit image of SDS-1. The satellite body is box-shaped, with two solar panels to be deployed in orbit. The SDS-1 will be launched in FY2008 as a piggyback payload on H-II A launch vehicle. The SDS-1 includes six missions, as described below.

- Multi-mode Integrated Transponder (MTP)

- Space-Wire demonstration Module (SWIM)

- Advanced Micro processing In-orbit experiment equipment (AMI)

- Thin Film Solar Cell (TFC)

- Small Dosimeter (DOS)

- Small satellite bus technology experiment

A mission of a total dose measurement system, DOS for the SDS-1 is a mission component and a means of support for other mission demonstration components and small satellite bus components by measurement of radiation tolerance. It consists of a RADFET Control Circuit (RCC) and seven sensors. One sensor contains an 8-pin LCC RADFET and a temperature sensor. Figure 8 depicts the system diagram of the total dose measurement system. The RCC consists of two printed boards and $146 \mathrm{~mm}$ wide $\times 25 \mathrm{~mm}$ high $\times 146 \mathrm{~mm}$ long aluminum chassis. The weight will be less than $0.6 \mathrm{~kg}$. The power consumption of the RCC is less than $2 \mathrm{~W}$. The RCC requires levels of $+5 \mathrm{~V}$. The telemetry data interfaces with Central Control Unit (CCU) at serial RS-422 protocol $^{9 \text { ). }}$.

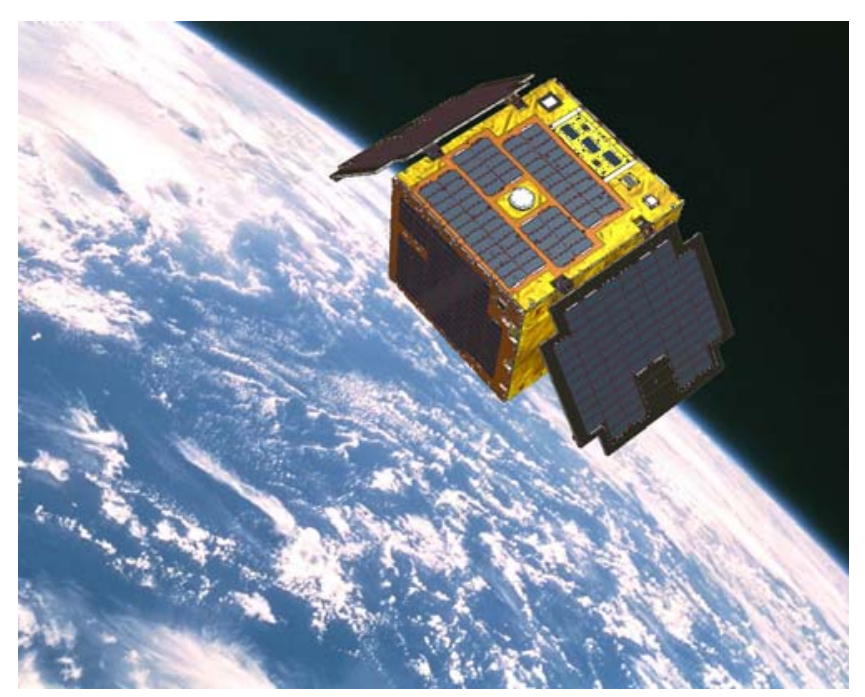

Fig. 7. On-orbit image of SDS-1. 


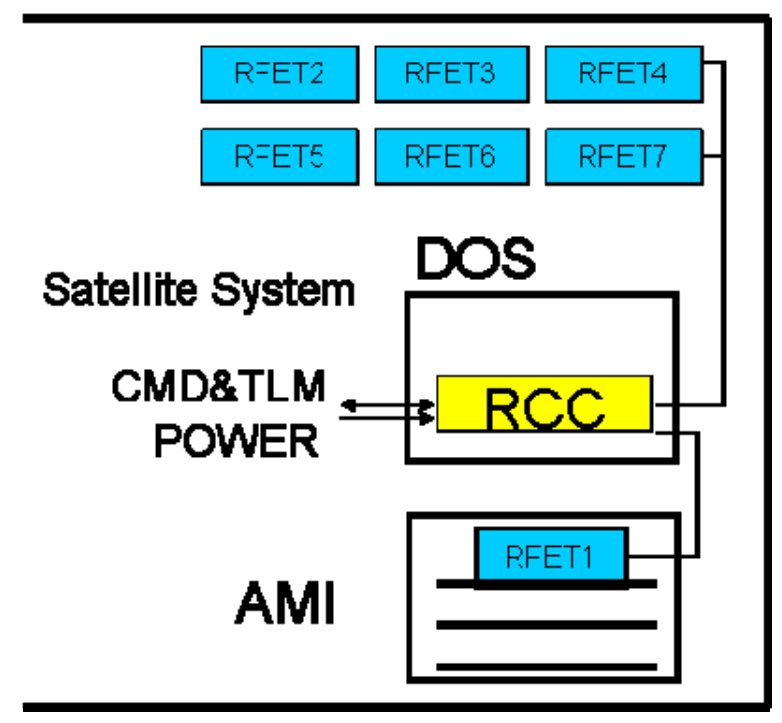

Fig. 8. System diagram of the total dose measurement system.

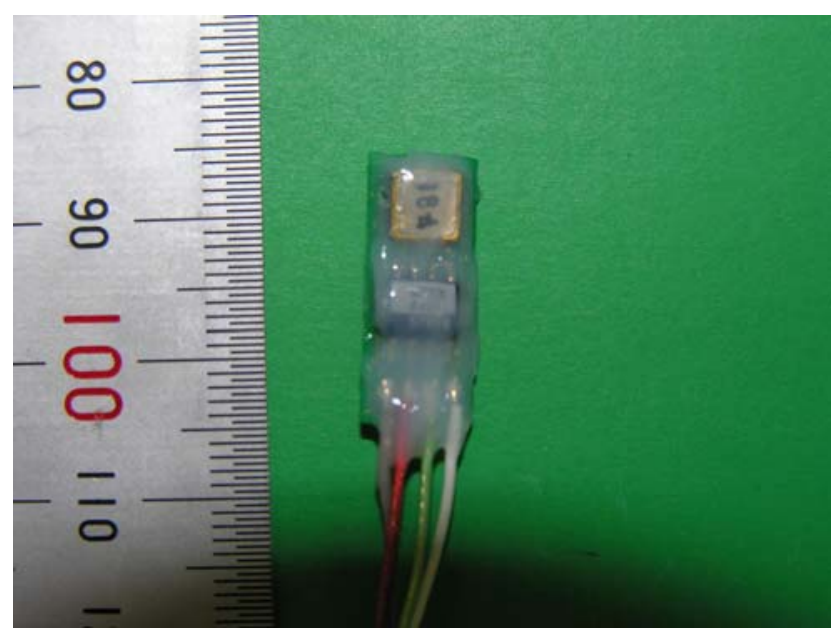

Fig. 9. Photograph of an RFET.

The RADFET used in SDS-1 is also a $400 \mathrm{~nm}$ implanted-gate oxide device manufactured by the Tyndall National Institute. We used the same RADFET for the SOHLA-1 mission. For the SDS-1 mission, the RADFET bare die is integrated to the 8-pin LCC package and arranged with a temperature sensor on a printed board. This integrated sensor is $8 \mathrm{~mm}$ wide $\times 3 \mathrm{~mm}$ high $\times 19 \mathrm{~mm}$ long and weighs approximately $4 \mathrm{~g}$ with $500 \mathrm{~mm}$ in its wire harness (Fig. 9). One sensor is mounted on a printed board in an AMI component. The other sensor will be attached to other mission demonstration components and small satellite bus components, as shown in Table 2 . The voltage $\left(V_{g s}\right)$ at the source with the drain/gate grounded is measured using a similar method as that for the SOHLA-1 satellite in Fig. 5.

Ground irradiation tests were conducted with the Co60 gamma-ray source. The RADFETs are as used in the RFET, but packaged in standard 14-lead Dual-in-Line package; they were irradiated at room temperature. This RADFET is the same lot of the RADFET used in the SOHLA-1 and SDS-1 mission. Measurements were done at certain levels using the computer-controlled parameter analyzer. The RADFETs were measured at $0.1,1,10,15,20,80$, and $200 \mathrm{~Gy}(\mathrm{Si})$.
Table 2. Location of the RFET in SDS-1.

\begin{tabular}{|l|l|}
\hline RFET number & Location \\
\hline 1 & Printed board in AMI \\
\hline 2 & Printed board in SWIM \\
\hline 3 & $\begin{array}{l}\text { Outside of Upper deck (This side always faces the } \\
\text { Sun.) }\end{array}$ \\
\hline 4 & $\begin{array}{l}\text { Inside of Lower deck (This side always faces } \\
\text { anti-Sun.) }\end{array}$ \\
\hline 5 & Inside of Side panel No. 2 \\
\hline 6 & Outside of Mission Access panel \\
\hline 7 & $\begin{array}{l}\text { Inside of Mission Access panel (This position is } \\
\text { close to MTP components.) }\end{array}$ \\
\hline
\end{tabular}

Figure 10 shows the $V_{g s}$ shift from the initial value $\left(d V_{g s}\right)$ with the total dose. Furthermore, 8 RADFETs were irradiated at each dose. It is noteworthy that variations between nominal samples were within 5\% for each dose. The annealing data at room temperature are shown in Fig. 11. These are data from other irradiation test runs for a different RADFET lot. After 1 Gy, 10 Gy, and 100 Gy irradiation, the $d V_{g s}$ respectively decreased by $5 \%, 1 \%$, and $20 \%$. These results indicate that the data should be correct at the $100 \mathrm{~Gy}$ absorbed dose level. The proton response of the RADFET, for example, in the $60 \mathrm{MeV}$ proton case, is lower by some $12-13 \%$ than the Co60 response ${ }^{10)}$. This indicates that the RADFET under thick shield thickness underestimates the total dose. The thickness is greater than $5 \mathrm{~mm}$ which the high-energy proton effect is dominant. This is also an error factor of measurement. This correction work is under investigation.

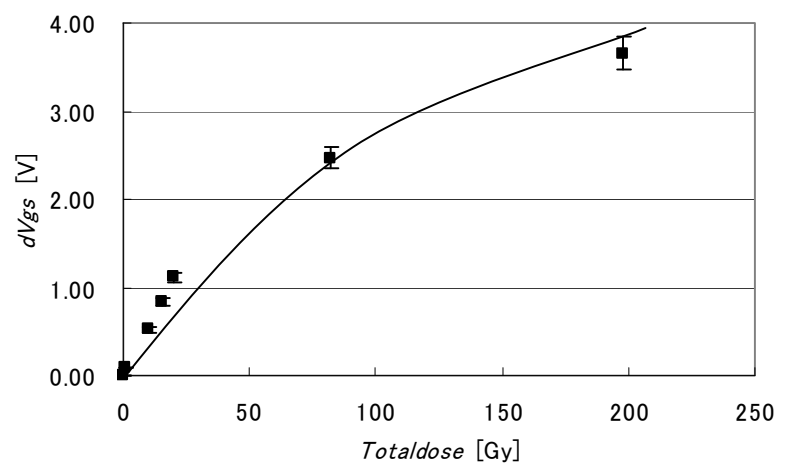

Fig. 10. Radiation response of the RADFETs. The $x$-axis shows the total dose [Gy ( $\mathrm{Si})]$ and the $y$-axis shows the difference of $V_{g s}$ from the initial value $\left(d V_{g s}\right)$. 


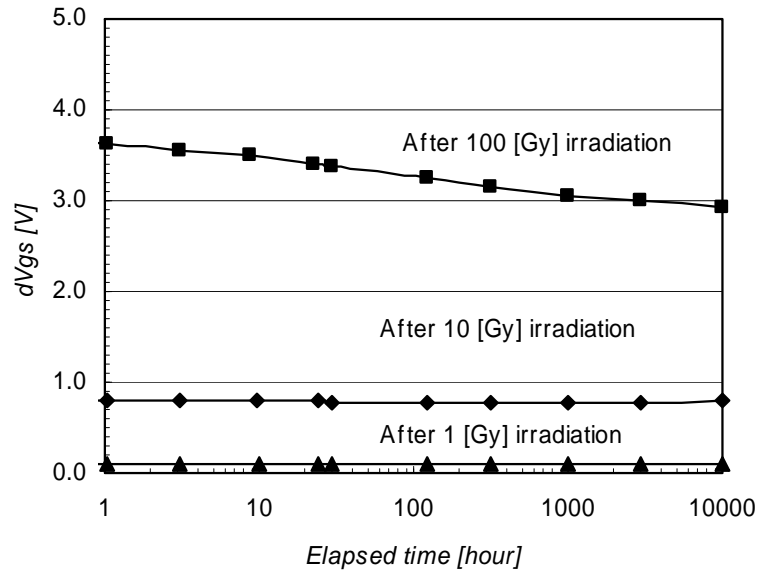

Fig. 11. Time dependence of $d V_{g s}$ fading data after irradiation (annealing data).

We analyzed the absorbed ionization dose using space radiation models and their effect codes. The models-AP8, AE8, and SHIELDOSE-2 models in SEES ${ }^{11)}$ - were used from 1 August 2008 to 1 August 2009. The target material was silicon. A solid sphere was used for shield geometry. The orbit was assumed to be a sun-synchronous, sub-recurrent orbit with an attitude of $700 \mathrm{~km}$ and an inclination of $98 \mathrm{deg}$. The absorbed dose at which a RFET is mounted was evaluated using FASTRAD. The output from SEES provides the source data for analyses. Figure 12 presents that the CAD image of the satellite wall holds the mission and bus components and the RFET in the AMI component.

The absorbed dose is $2 \mathrm{~Gy}(\mathrm{Si})$; the equivalent aluminum thickness information is, respectively, $2.77 \mathrm{~mm}, 182 \mathrm{~mm}$, minimum and maximum.

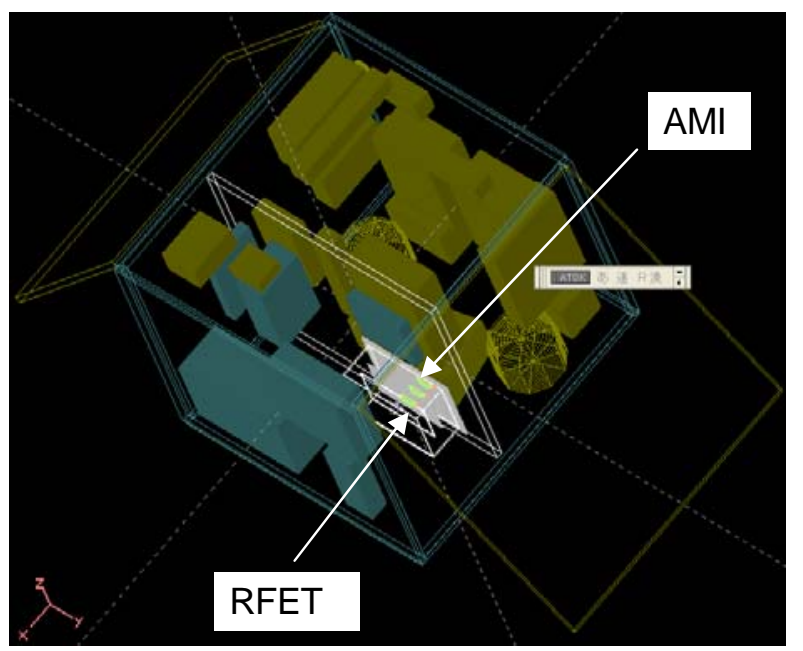

Fig. 12. CAD image of the SDS-1 structure and mission and bus components. Only the RFET in the AMI is analyzed.

Figure 13 depicts the total dose simulation results at three locations in Table 2. Only RFETs 1,5 , and 6 are calculated using $\mathrm{SEES}^{11)}$. In this case, the total dose time variation is calculated from 21 January 2008 to 21 January 2009. Because the locations have different shield thickness, the total dose is different from the positions which depend on the shield thickness. Furthermore, natural space environment conditions, which are affected by solar flare and radiation-belt particles, will dominate the total dose data.

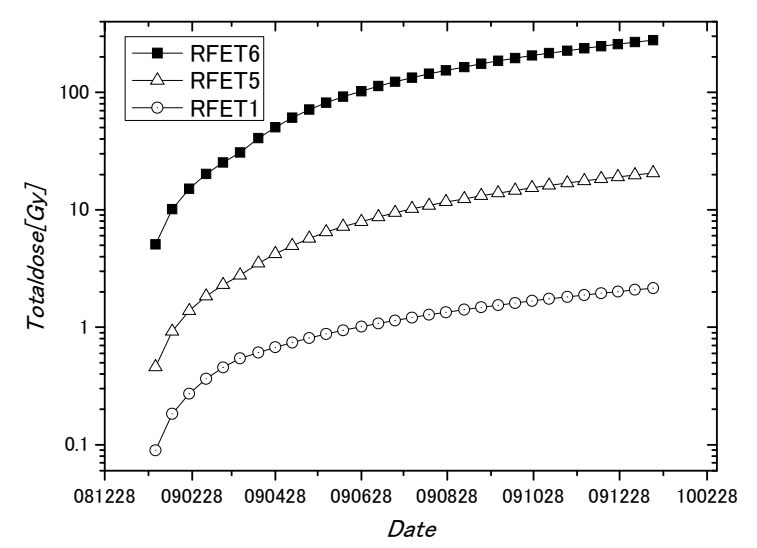

Fig. 13. Total dose time variation in RFET1, 5 and 6.

\section{Conclusion}

From satellite-borne monitors, JAXA has acquired in-flight space-environment data. A compact, total dose measurement system for the small satellites SOHLA-1 and SDS-1 has been developed based on a prior total ionizing dose effect measurement system. Particularly, the SDS-1 sensor is much smaller than the SOHLA-1 sensor. Seven RFETs are installed both inside SDS-1. The four RFETs are installed inside SOHLA-1. The absorbed ionizing dose where the RFETs are installed will be evaluated before flight for comparison with flight data.

\section{Acknowledgments}

The authors would like to thank Mr. Joe Mae of Nanotecs, Inc. for manufacturing two total dose measurement systems for SOHLA-1 and SDS-1. We appreciate the work of all people involved in the development the SOHLA-1 and SDS-1 projects. Especially, we would like to thank Dr. Yosuke Nakamura of the SDS-1 project team.

\section{References}

1) Yugo Kimoto, Hideki Koshiishi, Haruhisa Matsumoto, Tateo Goka, Aleksandar Jaksic: "Total dose effect measurement systems for spacecraft and the calibration method," Proc. of the 7th European Conference on Radiation and its effects on Components and systems, (2003), pp.621-626.

2) Tateo Goka, et al, "Space Environment \& Effect Measurements from the MDS-1 (Tsubasa) Satellite," Proc. of 23rd ISTS, (2002), pp.1747-1754

3) H. Koshiishi, H. Matsumoto, Y. Kimoto, H. Liu, T. Goka: "Space Environment Data Acquisition Equipment on Board Mission Demonstration Test Satellite," COSPAR Colloquia Series "Solar-Terrestrial Magnetic Activity and Space Environment" Edited by H. Wang, (2002), R. Xu, pp.369-371.

4) Yugo Kimoto, Hideki Koshiishi, Haruhisa Matsumoto, Tateo Goka: 
"Total Dose Orbital Data by Dosimeter Onboard Tsubasa (MDS-1) Satellite," IEEE Trans. Nucl. Sci. 50(6) (2003), pp.2301-2306.

5) Yugo Kimoto, Hiroshi Tachihara: "Small Total Dose Measurement System for Satellite", IAC-06-D5.2.03, Proc. of 57th IAC, (2006).

6) Yosuke Nakamura, Hidekazu Hashimoto: "SOHLA-1 - Low Cost Satellite Development with Technology Transfer Program of JAXA", IAC-05-B5.6.B.08, Proc. of 56th IAC, (2005).

7) T. Beutier, E. Delage, M. Wouts, O. Serres, P.-F. Peyrard: "FASTRAD New Tool for Radiation Prediction", Proc. of the 7th European Conference on Radiation and its effects on Components and systems, (2003), pp.181-183.

8) Yosuke Nakamura, Hiroaki Kawara, Keiichi Hirako, Hidekazu Hashimoto, "Small Demonstration Satellites in JAXA - Quick and Low-Cost System for Space Technology Advancement", Proc.of the 4S Symposium Small Satellites, Systems and Services, (2006).

9) Yugo Kimoto, Yohei Satoh, Hiroshi Tachihara: "Small Total Dose Measurement System for SDS-1", IAC-07-D5.2.02, Proc. of 58th IAC (2007).

10) Aleksandar Jaksic, Yugo Kimoto, Ali Mohammadzadeh, Wojtek Hajdas: "RADFET Response to Proton Irradiation under Different Biasing Configurations", IEEE Trans. Nucl. Sci. 53(4) (2006), pp.2004-2007.

11) http://sees.tksc.jaxa.jp/ 